\title{
ON A HIGHER-ORDER EVOLUTION EQUATION WITH A STEPANOV-BOUNDED SOLUTION
}

\author{
ARIBINDI SATYANARAYAN RAO
}

Received 12 June 2003 and in revised form 5 August 2004

\begin{abstract}
We study strong solutions $u: \mathbb{R} \rightarrow X$, a Banach space $X$, of the $n$ th-order evolution equation $u^{(n)}-A u^{(n-1)}=f$, an infinitesimal generator of a strongly continuous group $A$ : $D(A) \subseteq X \rightarrow X$, and a given forcing term $f: \mathbb{R} \rightarrow X$. It is shown that if $X$ is reflexive, $u$ and $u^{(n-1)}$ are Stepanov-bounded, and $f$ is Stepanov almost periodic, then $u$ and all derivatives $u^{\prime}, \ldots, u^{(n-1)}$ are strongly almost periodic. In the case of a general Banach space $X$, a corresponding result is obtained, proving weak almost periodicity of $u, u^{\prime}, \ldots, u^{(n-1)}$.
\end{abstract}

2000 Mathematics Subject Classification: 34G10, 34C27, 47D03.

1. Introduction. In this paper, we are concerned with an $n$ th-order evolution equation of the form

$$
u^{(n)}-A u^{(n-1)}=f
$$

Here $A: D(A) \subseteq X \rightarrow X$ is an infinitesimal generator of a strongly continuous group, $f: \mathbb{R} \rightarrow X$ a given forcing term, $X$ a Banach space with scalar field $C, n$ a positive integer, and $\mathbb{R}$ denotes the set of reals. We will give suitable assumptions to ensure that almost periodicity of the forcing term $f$ carries over to the solution $u$ and its derivatives up to order $(n-1)$.

The reason for studying this rather special evolution equation may be classified as a first pilot study of the issue of higher-order evolution equations, which probably has not been studied before.

We first recall the relevant concepts. A continuous function $f: \mathbb{R} \rightarrow X$ is said to be strongly (or Bochner) almost periodic if, for every given $\varepsilon>0$, there is an $r>0$ such that any interval in $\mathbb{R}$ of length $r$ contains a point $\tau$ for which

$$
\sup _{t \in \mathbb{R}}\|f(t+\tau)-f(t)\| \leq \varepsilon .
$$

Here $\|\cdot\|$ denotes the norm in $X$.

A function $f: \mathbb{R} \rightarrow X$ is called weakly almost periodic if $x^{*} f(\cdot): \mathbb{R} \rightarrow C$ is continuous and almost periodic for every $x^{*}$ in the dual space $X^{*}$ of $X$.

We will call a function $f \in L_{\text {loc }}^{1}(\mathbb{R}, X)$ Stepanov-bounded or briefly $S$-bounded if

$$
\|f\|_{S}:=\sup _{t \in \mathbb{R}} \int_{t}^{t+1}\|f(s)\| d s<\infty .
$$


We will call a function $f \in L_{\mathrm{loc}}^{1}(\mathbb{R}, X)$ Stepanov almost periodic or briefly $S$-almost periodic if, for every given $\varepsilon>0$, there is an $r>0$ such that any interval in $\mathbb{R}$ of length $r$ contains a point $\tau$ for which

$$
\sup _{t \in \mathbb{R}} \int_{t}^{t+1}\|f(s+\tau)-f(s)\| d s \leq \varepsilon
$$

We denote by $L(X, X)$ the set of all bounded linear operators on $X$ into itself. An operator-valued function $T: \mathbb{R} \rightarrow L(X, X)$ will be called a strongly continuous group if

$$
\begin{gathered}
T\left(t_{1}+t_{2}\right)=T\left(t_{1}\right) T\left(t_{2}\right) \quad \forall t_{1}, t_{2} \in \mathbb{R}, \\
T(0)=I=\text { the identity operator on } X, \\
T(\cdot) x: \mathbb{R} \rightarrow X \text { is continuous for every } x \in X .
\end{gathered}
$$

We recall (e.g., from Dunford and Schwartz [4]) that the infinitesimal generator $A$ : $D(A) \subseteq X \rightarrow X$ of a strongly continuous group $T: \mathbb{R} \rightarrow L(X, X)$ is a densely defined, closed linear operator.

An operator-valued function $T: \mathbb{R} \rightarrow L(X, X)$ is said to be strongly (weakly) almost periodic if $T(\cdot) x: \mathbb{R} \rightarrow X$ is strongly (weakly) almost periodic for every $x \in X$.

Suppose $A: D(A) \subseteq X \rightarrow X$ is a densely defined, closed linear operator, and $f: \mathbb{R} \rightarrow X$ is a continuous function. Then a strong solution of the evolution equation

$$
u^{(n)}(t)-A u^{(n-1)}(t)=f(t) \quad \text { a.e. for } t \in \mathbb{R}
$$

is an $n$ times strongly differentiable function $u: \mathbb{R} \rightarrow X$ with $u^{(n-1)}(t) \in D(A)$ for all $t \in \mathbb{R}$, and satisfies problem (1.8).

Our first result is as follows (see Zaidman $[7,8]$ for first-order evolution equations).

Theorem 1.1. Let $X$ be reflexive, $f: \mathbb{R} \rightarrow X$ continuous, $S$-almost periodic, A infinitesimal generator of a strongly almost periodic group $T: \mathbb{R} \rightarrow L(X, X)$. In this case, if, for the strong solution $u: \mathbb{R} \rightarrow X$ of problem (1.8), both $u$ and $u^{(n-1)}$ are $S$-bounded on $\mathbb{R}$, then $u, u^{\prime}, \ldots, u^{(n-1)}$ are all strongly almost periodic.

Our second result refers to a weak variant of our first theorem in the case of a general-not necessarily reflexive-Banach space $X$.

THEOREM 1.2. Suppose $f: \mathbb{R} \rightarrow X$ is an $S$-almost periodic (or a weakly almost periodic) continuous function, A an infinitesimal generator of a strongly continuous group $T: \mathbb{R} \rightarrow$ $L(X, X)$ such that the conjugate operator group $T^{*}: \mathbb{R} \rightarrow L\left(X^{*}, X^{*}\right)$ is strongly almost periodic. If, for the strong solution $u: \mathbb{R} \rightarrow X$ of problem (1.8), both $u$ and $u^{(n-1)}$ are $S$-bounded on $\mathbb{R}$, then $u, u^{\prime}, \ldots, u^{(n-1)}$ are all weakly almost periodic.

REMARK 1.3. For some examples of first-order and higher-order evolution equations with strongly almost periodic solutions, the reader may wish to consult Cooke [3] and Zaidman [9]. 


\section{Lemmas}

LEMMA 2.1. If $A$ is the infinitesimal generator of a strongly continuous group $G: \mathbb{R} \rightarrow$ $L(X, X)$, then the $(n-1)$ th derivative of any solution of $(1.8)$ has the representation

$$
u^{(n-1)}(t)=G(t) u^{(n-1)}(0)+\int_{0}^{t} G(t-s) f(s) d s \quad \text { for } t \in \mathbb{R} .
$$

Proof. For an arbitrary but fixed $t \in \mathbb{R}$, we have

$$
\begin{aligned}
\frac{d}{d s}\left[G(t-s) u^{(n-1)}(s)\right] & =G(t-s)\left[u^{(n)}(s)-A u^{(n-1)}(s)\right] \\
& =G(t-s) f(s) \text { a.e. for } s \in \mathbb{R} \text {, by }(1.8) .
\end{aligned}
$$

Now, integrating (2.2) from 0 to $t$, we obtain

$$
\int_{0}^{t} \frac{d}{d s}\left[G(t-s) u^{(n-1)}(s)\right] d s=\int_{0}^{t} G(t-s) f(s) d s,
$$

which gives the desired representation, by (1.6).

LEMMA 2.2. If $g: \mathbb{R} \rightarrow X$ is a strongly almost periodic function, and $G: \mathbb{R} \rightarrow L(X, X)$ is a strongly (weakly) almost periodic operator-valued function, then $G(\cdot) g(\cdot): \mathbb{R} \rightarrow X$ is a strongly (weakly) almost periodic function.

For the proof of Lemma 2.2, see [6, Theorem 1] for weak almost periodicity.

LEMмA 2.3. If $g: \mathbb{R} \rightarrow X$ is an $S$-almost periodic continuous function, and $G: \mathbb{R} \rightarrow$ $L(X, X)$ is a weakly almost periodic operator-valued function, then $x^{*} G(\cdot) g(\cdot): \mathbb{R} \rightarrow C$ is an $S$-almost periodic continuous function for every $x^{*} \in X^{*}$.

Proof. By our assumption, for an arbitrary but fixed $x^{*} \in X^{*}$, the function $x^{*} G(\cdot) x$ : $\mathbb{R} \rightarrow C$ is almost periodic, and so is bounded on $\mathbb{R}$, for every $x \in X$. Hence, by the uniform-boundedness principle,

$$
\sup _{t \in \mathbb{R}}\left\|x^{*} G(t)\right\|=K<\infty .
$$

We note that the function $x^{*} G(\cdot) g(\cdot)$ is continuous on $\mathbb{R}$ (see [6, proof of Theorem 1]).

Consider the functions $g_{\eta}$ given by

$$
g_{\eta}(t)=\frac{1}{\eta} \int_{0}^{\eta} g(t+s) d s \text { for } \eta>0, t \in \mathbb{R} .
$$

Since $g$ is $S$-almost periodic from $\mathbb{R}$ to $X, g_{\eta}$ is strongly almost periodic from $\mathbb{R}$ to $X$ for every fixed $\eta>0$. Further, as shown for $C$-valued functions in [2, pages 80-81], we can prove that $g_{\eta} \rightarrow g$ as $\eta \rightarrow 0+$ in the $S$-sense, that is,

$$
\sup _{t \in \mathbb{R}} \int_{t}^{t+1}\left\|g(s)-g_{\eta}(s)\right\| d s \longrightarrow 0 \quad \text { as } \eta \longrightarrow 0+.
$$

Now we have

$$
x^{*} G(s) g(s)=x^{*} G(s)\left[g(s)-g_{\eta}(s)\right]+x^{*} G(s) g_{\eta}(s) \quad \text { for } s \in \mathbb{R},
$$


and, by (2.4) and (2.6),

$$
\begin{aligned}
& \sup _{t \in \mathbb{R}} \int_{t}^{t+1}\left|x^{*} G(s)\left[g(s)-g_{\eta}(s)\right]\right| d s \\
& \quad \leq K \sup _{t \in \mathbb{R}} \int_{t}^{t+1}\left\|g(s)-g_{\eta}(s)\right\| d s \longrightarrow 0 \text { as } \eta \longrightarrow 0+.
\end{aligned}
$$

By Lemma 2.2, the functions $x^{*} G(\cdot) g_{\eta}(\cdot)$ are almost periodic from $\mathbb{R}$ to $C$. Therefore, it follows from (2.7)-(2.8) that $x^{*} G(\cdot) g(\cdot)$ is $S$-almost periodic from $\mathbb{R}$ to $C$.

LEMMA 2.4. If $g: \mathbb{R} \rightarrow X$ is an $S$-almost periodic continuous function, and $G: \mathbb{R} \rightarrow$ $L(X, X)$ is a strongly almost periodic operator-valued function, then $G(\cdot) g(\cdot): \mathbb{R} \rightarrow X$ is an $S$-almost periodic continuous function.

The proof of this lemma parallels that of Lemma 2.3 and may therefore be safely omitted.

LEMMA 2.5. In a reflexive space $X$, assume $h: \mathbb{R} \rightarrow X$ is an $S$-almost periodic continuous function, and

$$
H(t)=\int_{0}^{t} h(s) d s \quad \text { for } t \in \mathbb{R} .
$$

If $H$ is $S$-bounded, then it is strongly almost periodic from $\mathbb{R}$ to $X$.

For the proof of Lemma 2.5, see [5, Notes (ii)].

LEMмA 2.6. For an operator-valued function $G: \mathbb{R} \rightarrow L(X, X)$, suppose $G^{*}(t)$ is the conjugate (adjoint) of the operator $G(t)$ for $t \in \mathbb{R}$. If $G^{*}: \mathbb{R} \rightarrow L\left(X^{*}, X^{*}\right)$ is strongly almost periodic, and $g: \mathbb{R} \rightarrow X$ is weakly almost periodic, then $G(\cdot) g(\cdot): \mathbb{R} \rightarrow X$ is weakly almost periodic.

For the proof of Lemma 2.6, see [6, Remarks (iii)].

3. Proof of Theorem 1.1. By (2.1), we have

$$
T(-t) u^{(n-1)}(t)=u^{(n-1)}(0)+\int_{0}^{t} T(-s) f(s) d s \quad \text { for } t \in \mathbb{R} .
$$

Evidently, $T(-\cdot): \mathbb{R} \rightarrow L(X, X)$ is a strongly almost periodic group. Therefore, $T(-\cdot) x$ : $\mathbb{R} \rightarrow X$ is strongly almost periodic, and so is bounded on $\mathbb{R}$, for every $x \in X$. Hence, by the uniform-boundedness principle,

$$
\sup _{t \in \mathbb{R}}\|T(-t)\|<\infty .
$$

Consequently, $T(-\cdot) u^{(n-1)}(\cdot)$ is $S$-bounded on $\mathbb{R}$ (by our assumption, $u^{(n-1)}$ is $S$ bounded on $\mathbb{R}$ ).

Moreover, by Lemma $2.4, T(-\cdot) f(\cdot): \mathbb{R} \rightarrow X$ is an $S$-almost periodic continuous function. So, by Lemma $2.5, T(-\cdot) u^{(n-1)}(\cdot)$ is strongly almost periodic from $\mathbb{R}$ to $X$. Hence, by Lemma $2.2, u^{(n-1)}(\cdot)=T(\cdot)\left[T(-\cdot) u^{(n-1)}(\cdot)\right]$ is strongly almost periodic from $\mathbb{R}$ to $X$. 
Now consider a sequence $\left(\alpha_{k}\right)_{k=1,2, \ldots}$ of infinitely differentiable nonnegative functions on $\mathbb{R}$ such that

$$
\alpha_{k}(t)=0 \quad \text { for }|t| \geq \frac{1}{k}, \quad \int_{-1 / k}^{1 / k} \alpha_{k}(t) d t=1 .
$$

The convolution of $u$ and $\alpha_{k}$ is defined by

$$
\left(u^{*} \alpha_{k}\right)(t)=\int_{\mathbb{R}} u(t-s) \alpha_{k}(s) d s=\int_{\mathbb{R}} u(s) \alpha_{k}(t-s) d s \quad \text { for } t \in \mathbb{R} .
$$

We set

$$
C_{\alpha_{k}}=\max _{|t| \leq 1 / k} \alpha_{k}(t)
$$

Then we have

$$
\begin{aligned}
\left\|\left(u^{*} \alpha_{k}\right)(t)\right\| & =\left\|\int_{-1}^{1} u(t-s) \alpha_{k}(s) d s\right\| \leq C_{\alpha_{k}} \int_{t-1}^{t+1}\|u(\rho)\| d \rho \\
& \leq 2 C_{\alpha_{k}}\|u\|_{S} \quad \text { for } t \in \mathbb{R} \text {, by }(1.3) .
\end{aligned}
$$

That is, $u^{*} \alpha_{k}$ is bounded on $\mathbb{R}$.

We note that, for $m=1,2, \ldots, n-1$ and $k=1,2, \ldots$,

$$
\left(u^{*} \alpha_{k}\right)^{(m)}(t)=\left(u^{(m) *} \alpha_{k}\right)(t) \text { for } t \in \mathbb{R} .
$$

Further, since $u^{(n-1)}$ is strongly almost periodic from $\mathbb{R}$ to $X,\left(u^{*} \alpha_{k}\right)^{(n-1)}=\left(u^{(n-1) *} \alpha_{k}\right)$ is strongly almost periodic from $\mathbb{R}$ to $X$. Consequently, by [3, corollary to Lemma 5], $u^{*} \alpha_{k}, u^{\prime *} \alpha_{k}, \ldots, u^{(n-2) *} \alpha_{k}$ are all strongly almost periodic from $\mathbb{R}$ to $X$.

With $u^{(n-1)}$ being bounded on $\mathbb{R}, u^{(n-2)}$ is uniformly continuous on $\mathbb{R}$. Therefore, the sequence of convolutions $\left(u^{(n-2) *} \alpha_{k}\right)(t) \rightarrow u^{(n-2)}(t)$ as $k \rightarrow \infty$, uniformly for $t \in \mathbb{R}$. Hence $u^{(n-2)}$ is strongly almost periodic from $\mathbb{R}$ to $X$. We thus conclude successively that $u^{(n-2)}, \ldots, u^{\prime}, u$ are all strongly almost periodic from $\mathbb{R}$ to $X$, completing the proof of the theorem.

4. Proof of Theorem 1.2. By our assumption, for an arbitrary but fixed $x^{*} \in X^{*}$, $x^{*} T(\cdot)=T^{*}(\cdot) x^{*}: \mathbb{R} \rightarrow X^{*}$ is strongly almost periodic, and so $x^{*} T(\cdot) x: \mathbb{R} \rightarrow C$ is almost periodic for every $x \in X$. Therefore, it follows that $T: \mathbb{R} \rightarrow L(X, X)$ is a weakly almost periodic group.

By (3.1), we have

$$
x^{*} T(-t) u^{(n-1)}(t)=x^{*} u^{(n-1)}(0)+\int_{0}^{t} x^{*} T(-s) f(s) d s \quad \text { for } t \in \mathbb{R} .
$$

By Lemma 2.3, $x^{*} T(-\cdot) f(\cdot): \mathbb{R} \rightarrow C$ is an $S$-almost periodic continuous function. By (2.4), $x^{*} T(-\cdot) u^{(n-1)}(\cdot)$ is $S$-bounded on $\mathbb{R}$, and so, by Lemma 2.5 , is almost periodic from $\mathbb{R}$ to $C$. That is, $T(-\cdot) u^{(n-1)}(\cdot)$ is weakly almost periodic from $\mathbb{R}$ to $X$. Consequently, by Lemma $2.6, u^{(n-1)}(\cdot)=T(\cdot)\left[T(-\cdot) u^{(n-1)}(\cdot)\right]$ is weakly almost periodic from $\mathbb{R}$ to $X$. 
For the sequence $\left(\alpha_{k}\right)_{k=1,2, \ldots}$ defined by (3.3), $\left(x^{*} u^{*} \alpha_{k}\right)=x^{*}\left(u^{*} \alpha_{k}\right)$ is bounded on $\mathbb{R}$ (by (3.6)). Further, for $m=1,2, \ldots, n-1$ and $k=1,2, \ldots$, we have

$$
\left(x^{*} u^{*} \alpha_{k}\right)^{(m)}(t)=\left(x^{*} u^{(m) *} \alpha_{k}\right)(t) \text { for } t \in \mathbb{R} .
$$

Now the rest of the proof is obvious.

If $f: \mathbb{R} \rightarrow X$ is weakly almost periodic, then by Lemma 2.6, $T(-\cdot) f(\cdot): \mathbb{R} \rightarrow X$ is weakly almost periodic.

REMARK 4.1. If $T(t) \equiv I$ for $t \in \mathbb{R}$, and so $A=0$, then problem (1.8) reduces to

$$
u^{(n)}(t)=f(t) \quad \text { a.e. for } t \in \mathbb{R} \text {. }
$$

(i) In a reflexive space $X$, suppose $f$ is defined as in Theorem 1.1. If $u: \mathbb{R} \rightarrow X$ is an $S$ bounded strong solution of problem (4.3), then $u, u^{\prime}, \ldots, u^{(n-1)}$ are all strongly almost periodic from $\mathbb{R}$ to $X$.

(ii) Assume $f: \mathbb{R} \rightarrow X$ is a weakly almost periodic continuous function. If $u: \mathbb{R} \rightarrow X$ is an $S$-bounded strong solution of problem (4.3), then $u, u^{\prime}, \ldots, u^{(n-1)}$ are all weakly almost periodic from $\mathbb{R}$ to $X$.

These statements are clearly special cases of Theorems 1.1 and 1.2 if we take into account that the assumption $u^{(n-1)} S$-bounded can be omitted, since, by (4.3), $u^{(n)}$ is $S$-almost periodic, and so $u^{(n-1)}$ is strongly (weakly) uniformly continuous on $\mathbb{R}$ (by Amerio and Prouse [1, Theorem 8, page 79]).

\section{REFERENCES}

[1] L. Amerio and G. Prouse, Almost-Periodic Functions and Functional Equations, Van Nostrand Reinhold, New York, 1971.

[2] A. S. Besicovitch, Almost Periodic Functions, Dover Publications, New York, 1955.

[3] R. Cooke, Almost periodicity of bounded and compact solutions of differential equations, Duke Math. J. 36 (1969), 273-276.

[4] N. Dunford and J. T. Schwartz, Linear Operators. I. General Theory, Pure and Applied Mathematics, vol. 7, Interscience Publishers, New York, 1958.

[5] A. S. Rao, On differential operators with Bohr-Neugebauer type property, J. Differential Equations 13 (1973), 490-494.

[6] On weakly almost-periodic families of linear operators, Canad. Math. Bull. 18 (1975), no. $1,81-85$.

[7] S. Zaidman, Some asymptotic theorems for abstract differential equations, Proc. Amer. Math. Soc. 25 (1970), 521-525.

[8] - Some remarks on almost-periodicity, Atti Accad. Sci. Torino Cl. Sci. Fis. Mat. Natur. 106 (1972), 63-67.

[9] _ Abstract differential equations with almost-periodic solutions, J. Math. Anal. Appl. 107 (1985), no. 1, 291-299.

Aribindi Satyanarayan Rao: Department of Computer Science, Vanier College, 821 Avenue Ste Croix, St. Laurent, Quebec, Canada H4L 3X9

E-mail address: aribindr@vani ercol1ege.qc.ca 


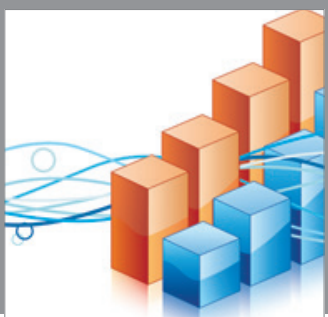

Advances in

Operations Research

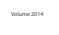

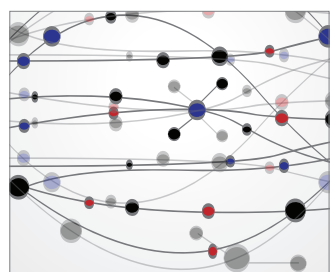

\section{The Scientific} World Journal
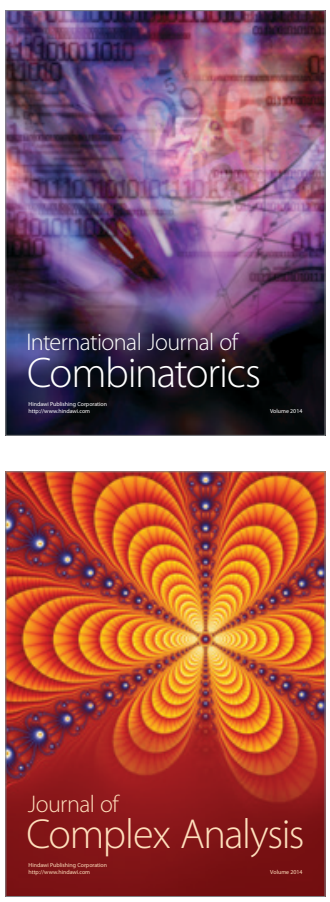

International Journal of

Mathematics and

Mathematical

Sciences
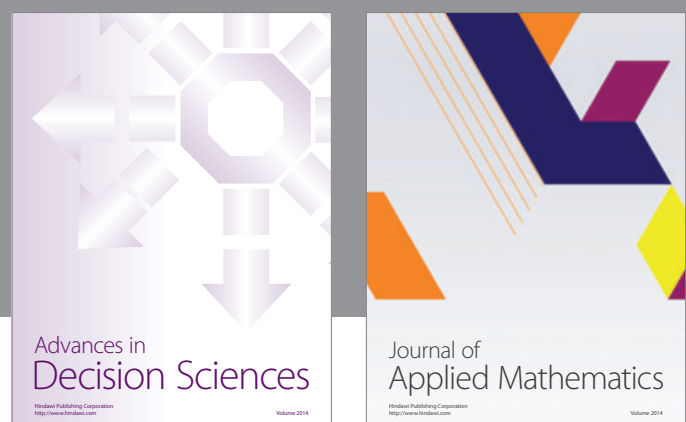

Journal of

Applied Mathematics
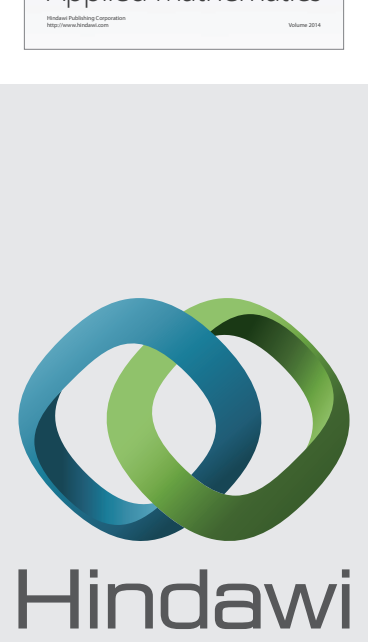

Submit your manuscripts at http://www.hindawi.com
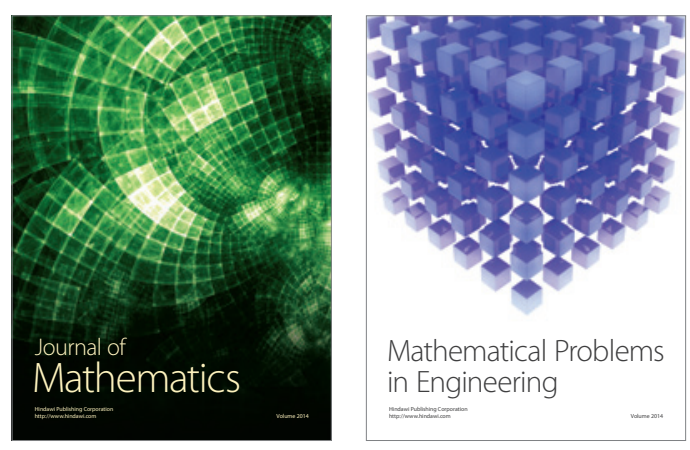

Mathematical Problems in Engineering
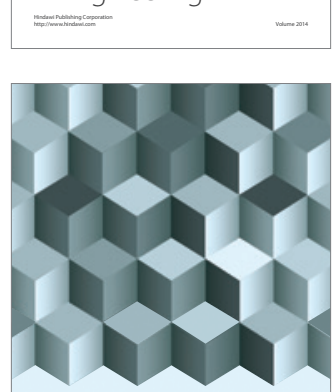

Journal of

Function Spaces
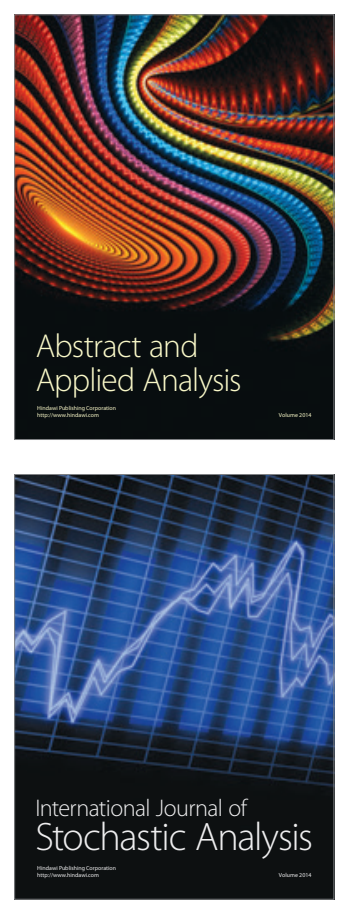

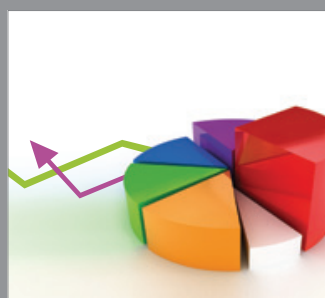

ournal of

Probability and Statistics

Promensencen
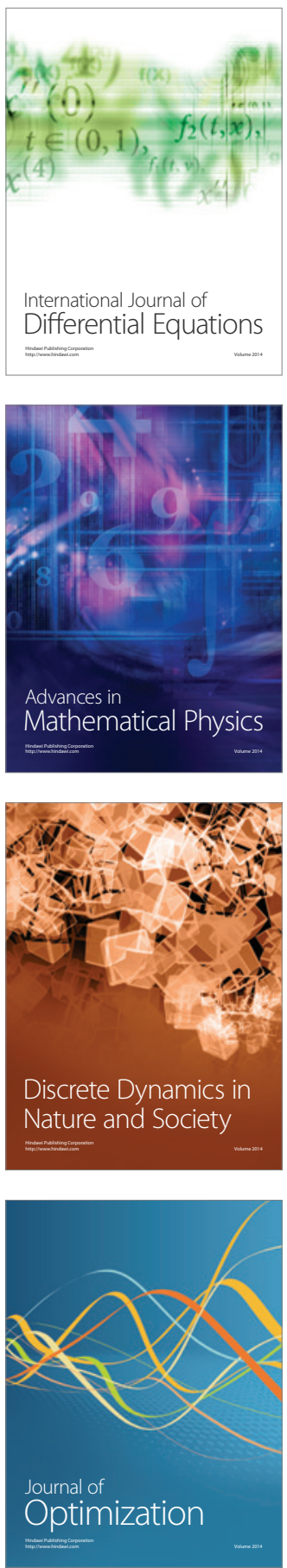